PROCEEDINGS OF THE

AMERICAN MATHEMATICAL SOCIETY

Volume 133, Number 4, Pages 1185-1195

S 0002-9939(04)07807-4

Article electronically published on November 1, 2004

\title{
K-THEORY TOOLS FOR LOCAL AND ASYMPTOTIC CYCLIC COHOMOLOGY
}

\author{
VAHID SHIRBISHEH
}

(Communicated by David R. Larson)

\begin{abstract}
A generalization of the Connes-Thom isomorphism is given for stable, homotopy invariant, and split exact functors on separable $C^{*}$-algebras. As examples of these functors, we concentrate on asymptotic and local cyclic cohomology, and the result is applied to improve some formulas in asymptotic and local cyclic cohomology of $C^{*}$-algebras. As another application, it is shown that these cyclic theories are rigid under Rieffel's deformation quantizations.
\end{abstract}

\section{INTRODUCTION}

Motivation for this paper comes from two sources. The first is [R4, A2, where it was proved that $K$-theory is rigid under Rieffel's deformation quantizations. The second is Rosenberg's works, Ro1, Ro2. In [Ro1, Rosenberg studied the behavior of algebraic $K$-theory under formal deformation quantization. In Ro2], he discussed the relations between $K$-theory and various quantization theories, and he suggested a similar study on Connes' cyclic homology. As cyclic homology of formal deformation quantization has been studied by Nest and Tsygan in [NT1, NT2, it is natural to ask how cyclic (co)homology behaves under other quantization theories. Because of the algebraic nature of Connes' cyclic theory, we do not hope to get any satisfactory answer to this question on $C^{*}$-algebraic quantizations. Therefore, in such situations, we have to use other cyclic theories that work like Connes' cyclic theory and are appropriate for dealing with topological algebras.

Bivariant local cyclic cohomology of admissible Fréchet algebras with compact supports and bivariant asymptotic cyclic cohomology of admissible Fréchet algebras are such theories; see [Pu1, Pu2, $\mathrm{Pu} 3$. On commutative $C^{*}$-algebras, they are comparable with the cohomology of the character spaces as locally compact topological spaces. Moreover, they are stable and homotopy invariant bifunctors which appropriate excisions hold in both their variables, and there are bivariant Chern-Connes characters from $K K$-theory into them.

Among $C^{*}$-algebraic quantization theories, Rieffel's deformation quantizations, [R1, R3], are the best theories for our purpose, because they are associated appropriately to crossed product $C^{*}$-algebras (up to strong Morita equivalence), [R4, A2], and the set of their examples contains several important noncommutative spaces.

Received by the editors March 26, 2002 and, in revised form, December 10, 2003.

2000 Mathematics Subject Classification. Primary 46L80; Secondary 46L65.

Key words and phrases. KK-theory, $C^{*}$-crossed product, local and asymptotic cyclic cohomology, excision, strong Morita equivalence, Rieffel's deformation quantizations.

(C)2004 American Mathematical Society Reverts to public domain 28 years from publication 
It is clear from [R4, A2 that in order to repeat their proofs for other functors, we need Morita invariance and the Connes-Thom isomorphism for them. So, we provide these tools in section 1 . Section 2 is devoted to the application of these tools in local and specially asymptotic cyclic cohomology, and we state an unstable excision for bivariant asymptotic cyclic cohomology. In section 3 we show that local and asymptotic cyclic (co)homology groups of noncommutative and commutative Heisenberg manifolds are isomorphic. As another example of strict deformation quantization, we prove rigidity of local and asymptotic cyclic theories under deformation quantization by actions of $\mathbb{R}^{n}$.

In this paper, we restrict ourselves to separable $C^{*}$-algebras and their dense *-subalgebras. $\mathcal{K}$ denotes the $C^{*}$-algebra of compact operators on an infinitedimensional separable Hilbert space. The functor $S-$ is suspension, i.e., for any $C^{*}$-algebra $A, S A=A \otimes C_{0}(\mathbb{R})$. Let $F$ be a functor on the category of $C^{*}$-algebras. $F$ is called stable if for every $C^{*}$-algebra $A$ the natural embedding $A \rightarrow A \otimes \mathcal{K}$ induces a natural isomorphism $F(A) \rightarrow F(A \otimes \mathcal{K}), F$ is called homotopy invariant if $F(f)=F(g): F(A) \rightarrow F(B)$, whenever $f, g \in H o m(A, B)$ are homotopic and $F$ is called split exact when for every split exact sequence $0 \rightarrow A \stackrel{f}{\rightarrow} B \stackrel{p}{\rightarrow} C \rightarrow 0$ with splitting *-homomorphism $g: C \rightarrow B$, the map $F(f) \oplus F(g): F(A) \oplus F(C) \rightarrow F(B)$ is an isomorphism.

\section{Connes-Thom ISOMORPHISM}

We use some techniques of $K K$-theory to prove the Connes-Thom isomorphism. Our approach is known as Cuntz's picture of $K K$-theory, C3. We note that Bott periodicity and the Pimsner-Voiculescu exact sequence were previously studied in the same way as in C2 and our proof for Theorem 1.2 is an application of the Fack and Skandalis result, [FS].

A quasihomomorphism between two $C^{*}$-algebras $A$ and $B$ is a diagram as follows:

$$
A \underset{\bar{\alpha}}{\stackrel{\alpha}{\rightrightarrows}} E \triangleright J \stackrel{\mu}{\rightarrow} B
$$

where $E$ and $J$ are $C^{*}$-algebras, $J \triangleleft E$, and $\alpha, \bar{\alpha}, \mu$ are ${ }^{*}$-homomorphisms such that

(i) $\mu$ is an inclusion,

(ii) $E$ is the $C^{*}$-algebras generated by $\alpha(A)$ and $\bar{\alpha}(A)$,

(iii) $J$ is the closed two-sided ideal generated by $\alpha(x)-\bar{\alpha}(x), x \in A$ in $E$,

(iv) the composition of $\alpha$ and the quotient map $E \rightarrow E / J$ is injective, thus, an isomorphism.

(A diagram as above with only the property $\alpha(x)-\bar{\alpha}(x) \in J$, for $x \in A$ is called a prequasihomomorphism.) Now, letting $A$ be a $C^{*}$-algebra, by definition, $Q A$ is the universal *-algebra generated by symbols $x, q(x), x \in A$, satisfying the relation $q(x y)=x q(y)+q(x) y-q(x) q(y)$, and let $q A$ be defined as the ideal generated by $q(x), x \in A$ in $Q A$. We equip $Q A$ with the largest $C^{*}$-norm,

$$
\|x\|_{\infty}=\sup \left\{\|\pi(x)\| ; \pi \text { is a }{ }^{*} \text {-representation }\right\}
$$

and still write $Q A, q A$ for the completions of $Q A, q A$ with respect to this norm.

For two $C^{*}$-algebras $A$ and $B$, let $[q A, B \otimes \mathcal{K}]$ be the set of homotopy classes of *-homomorphisms from $q A$ to $B \otimes \mathcal{K}$. On this set, addition is defined by $[\varphi]+[\psi]=$ 
$\left[\left(\begin{array}{ll}\varphi & 0 \\ 0 & \psi\end{array}\right)\right]$. By this addition it becomes an Abelian group equal to $K K(A, B)$ (or more exactly, $K K_{0}(A, B)$ ); for details, see [C1, C3].

We have two homomorphisms $\imath, \bar{\imath}$ from $A$ into $Q A$ defined by $\imath(x)=x, \bar{\imath}=$ $x-q(x)$. The quasihomomorphism given by the diagram

$$
A \underset{\bar{\imath}}{\stackrel{\imath}{\rightrightarrows}} Q A \triangleright q A
$$

is the universal quasihomomorphism from $A$ into $q A$. Let $\varphi: q A \rightarrow B$ be a ${ }^{*}$ homomorphism. $\varphi$ is naturally associated to the quasihomomorphism

$$
A \underset{\pi \bar{\imath}}{\stackrel{\pi \imath}{\rightrightarrows}} Q A / \operatorname{ker} \varphi \triangleright q A / \operatorname{ker} \varphi \stackrel{\bar{\varphi}}{\rightarrow} B
$$

where $\pi: Q A \rightarrow Q A / \operatorname{ker} \varphi$ and $\bar{\varphi}: q A / \operatorname{ker} \varphi \rightarrow B$ are, respectively, the quotient map, and the inclusion map defined by $\varphi$. (and vice versa, every quasihomomorphism from $A$ to $B$ gives rise to a homomorphism from $q A$ to $B$; for details, see Proposition 1.1 of [C3]).

The following result is the main tool in our study. It was stated for functors from the category of $C^{*}$-algebras to the category of $\mathbb{Z}$-modules in [C3], and can be restated for any commutative ring $R$, instead of $\mathbb{Z}$.

Proposition 1.1 (Cuntz, [C3, 2.2.a]). Let $F$ be a stable, homotopy invariant, split exact functor from the category of $C^{*}$-algebras to that of $R$-modules. Every $\varphi \in K K(A, B)$ induces a morphism $F(\varphi): F(A) \rightarrow F(B)$ compatible with the Kasparov product, i.e. $F(\psi \varphi)=F(\psi) F(\varphi)$ for any $\psi \in K K(B, C)$.

Proof. We sketch only the definition of $F(\varphi)$. Consider $\varphi$ as a ${ }^{*}$-homomorphism from $q A$ to $B$. As we saw, this ${ }^{*}$-homomorphism is associated to the quasihomomorphism

$$
A \underset{\pi \bar{\imath}}{\stackrel{\pi \imath}{\rightrightarrows}} Q A / \operatorname{ker} \varphi \triangleright q A / \operatorname{ker} \varphi \stackrel{\bar{\varphi}}{\rightarrow} B
$$

and this quasihomomorphism induces the desired morphism as $F(\varphi)=F(\bar{\varphi})(F(\pi \imath)$ $-F(\pi \bar{\imath}))$. According to the definition, it is obvious that $F(\varphi)(r-)=r F(\varphi)(-)$ for $r \in R$.

The above result shows that in order to obtain a desired morphism between $F(A)$ and $F(B)$ it is enough to find an appropriate element of $K K(A, B)$. An element of $K K(A, B)$ inducing an isomorphism is called $K K$-equivalence. Equivalently, $\mathbf{x} \in K K(A, B)$ is a $K K$-equivalence if there is $\mathbf{y} \in K K(B, A)$ such that $\mathbf{x y}=$ $1_{B}, \mathbf{y x}=1_{A}$. If there is a $K K$-equivalence in $K K(A, B), A$ and $B$ are called $K K$-equivalent.

In [C2 Bott periodicity and the Pimsner-Voiculescu exact sequence were constructed for functors described in 1.1. Now the Connes-Thom isomorphism is accessible by applying the $K K$-equivalence $\mathbf{t}_{\alpha} \in K K\left(A \times_{\alpha} \mathbb{R}, S A\right)$ set forth in section 19.3 of $[\mathrm{B}]$.

Theorem 1.2. Let $F$ be a functor such as in 1.1, and let $\alpha$ be a (strongly) continuous action of $\mathbb{R}$ on a $C^{*}$-algebra $A$. Then $F\left(\mathbf{t}_{\alpha}\right)$ is an isomorphism between $F\left(A \times_{\alpha} \mathbb{R}\right)$ and $F(S A)$.

Remark 1.3. Let $\alpha$ be an automorphism of a $C^{*}$-algebra $A$. As was done in [C2], the Pimsner-Voiculescu exact sequence can be constructed using a $K K$-equivalence 
in $K K\left(A, T_{\alpha}\right)$, where $T_{\alpha}$ is the Toeplitz algebra associated to the automorphism $\alpha$. This $K K$-equivalence allows us to replace $K$-groups of the Toeplitz algebra with $K$ groups of $A$ in the six-term exact sequence associated to the following short exact sequence, known as the Toeplitz extension:

$$
0 \rightarrow \mathcal{K} \otimes A \rightarrow T_{\alpha} \rightarrow A \times_{\alpha} \mathbb{Z} \rightarrow 0
$$

Therefore, in order to obtain the P-V exact sequence for a functor, only the conditions assumed in 1.1 are necessary.

Since Pimsner's and Voiculescu's paper, [PV], has appeared, their work has found several generalizations, $\mathrm{E} \mathrm{KhS}, \mathrm{P} \mathrm{AEE}$. In all of them $A \times_{\alpha} \mathbb{Z}$ is replaced by a new $C^{*}$-algebra, e.g. $A \overline{\times}_{E} \mathbb{Z}$, which generalizes the crossed product of the $C^{*}$-algebra $A$ by $\mathbb{Z}$, and we have a generalized Toeplitz extension of $A$ by a generalized Toeplitz algebra, e.g. $\bar{T}_{E}$, which is $K K$-equivalent to $A$. The six-term exact sequences of such extensions are considered as generalizations of the P-V exact sequence. One can repeat our discussion for these generalizations.

Let $\alpha$ be an automorphism of a $C^{*}$-algebra $A$. The mapping torus of $\alpha$ is

$$
M_{\alpha}=\{f:[0,1] \rightarrow A ; f(1)=\alpha(f(0))\} .
$$

Consider the action of $\mathbb{Z}$ on $A$ defined by $\alpha$. Its dual $\hat{\alpha}$ is an action of the dual of $\mathbb{Z}, \mathbb{T}=\hat{\mathbb{Z}}$, on $A \times_{\alpha} \mathbb{Z}$. Let $\pi: \mathbb{R} \rightarrow \mathbb{R} / \mathbb{Z}$ be the quotient map. Then $\alpha^{\prime}=\hat{\alpha} o \pi$ is an action of $\mathbb{R}$ on $A \times_{\alpha} \mathbb{Z}$, which is trivial on $\mathbb{Z}$ and $A \times_{\alpha} \mathbb{Z} \times{ }_{\alpha^{\prime}} \mathbb{R}$ is isomorphic to the mapping torus of $\hat{\hat{\alpha}}$ on $A \times_{\alpha} \mathbb{Z} \times_{\hat{\alpha}} \mathbb{T}$ (see $[\mathrm{B}$, Proposition 10.3.2). By Takai duality, this means $A \times{ }_{\alpha} \mathbb{Z} \times{ }_{\alpha^{\prime}} \mathbb{R}$ is isomorphic to the mapping torus of $\alpha \otimes A d_{\rho}$ on $A \otimes \mathcal{K}$, where $\rho$ is the right regular representation of $\mathbb{Z}$ and $\mathcal{K}$ is thought of as the $C^{*}$-algebra of compact operators on $\ell^{2}(\mathbb{Z})$. Now, suppose $\alpha, \beta$ are two homotopic automorphisms of $A$. Then $\alpha \otimes A d_{\rho}$ and $\beta \otimes A d_{\rho}$ are homotopic too. Applying Proposition 10.5.1 of [B] we deduce that $A \times{ }_{\alpha} \mathbb{Z} \times{ }_{\alpha^{\prime}} \mathbb{R}$ is isomorphic to $A \times{ }_{\beta} \mathbb{Z} \times{ }_{\beta^{\prime}} \mathbb{R}$. Thus, as a generalization of Corollary 10.5.2 of [B], we have the following result:

Corollary 1.4. Let $F$ be a functor as in 1.1 and let $\alpha, \beta$ be two homotopic automorphisms of a $C^{*}$-algebra $A$. Then $F\left(A \times_{\alpha} \mathbb{Z}\right) \cong F\left(A \times_{\beta} \mathbb{Z}\right)$.

Morita invariance. It is well known that two strongly Morita equivalent $\sigma$-unital $C^{*}$-algebras are stably isomorphic, $\left[\mathrm{BGR}\right.$. Since every separable $C^{*}$-algebra is $\sigma$-unital and because of our assumptions about functors, we have:

Remark 1.5. In our discussion, all functors have the strong Morita invariance property on separable $C^{*}$-algebras.

\section{LOCAL AND ASYMPTOTIC CYCLIC COHOMOLOGY}

Asymptotic and local cyclic cohomology are stable in both variables (see Theorem 8.18 of [Pu1] and Corollary 4.10 of [Pu2]). Also, composition products and continuous homotopy theorems prove their homotopy invariances (see Theorems 6.5 and 6.15 of [Pu1] and Theorems 3.5 and 3.18 of [Pu2]). But, there is a difference between local cyclic theory and asymptotic cyclic theory about split exactness. First we study asymptotic cyclic cohomology. 
2.1. Asymptotic cyclic cohomology. At the moment, we have only stable excisions for asymptotic cyclic homology and cohomology, Theorem 9.9 of [Pu1]; that is, for a split short exact sequence of $C^{*}$-algebras, $0 \rightarrow I \rightarrow A \rightarrow B \rightarrow 0$, associated six-term exact sequences give rise to four split short exact sequences as follows:

$$
\begin{aligned}
& 0 \rightarrow H C_{\alpha}^{*}(C, S I) \rightarrow H C_{\alpha}^{*}(C, S A) \rightarrow H C_{\alpha}^{*}(C, S B) \rightarrow 0, *=0,1, \\
& 0 \rightarrow H C_{\alpha}^{*}(S B, C) \rightarrow H C_{\alpha}^{*}(S A, C) \rightarrow H C_{\alpha}^{*}(S I, C) \rightarrow 0, *=0,1 .
\end{aligned}
$$

Therefore, for any $C^{*}$-algebra $C$, the functor $H C_{\alpha}^{*}(C, S-)$ and the cofunctor $H C_{\alpha}^{*}(S-, C)$ are split exact. In order to get unstable split exactness for asymptotic cyclic theory, we need an unstable excision.

Theorem 2.1. For a given admissible Fréchet algebra $C$, any extension of admissible Fréchet algebras

$$
0 \rightarrow I \stackrel{i}{\rightarrow} A \underset{f}{\stackrel{s}{\leftrightarrows}} B \rightarrow 0
$$

with a bounded linear section $s: B \rightarrow A$ induces the following six-term exact sequences:

and

$$
\begin{array}{ccccc}
H C_{\alpha}^{0}(C, I) & \longrightarrow & H C_{\alpha}^{0}(C, A) & \stackrel{f_{*}}{\longrightarrow} & H C_{\alpha}^{0}(C, B) \\
\uparrow \partial & & & \downarrow \partial \\
H C_{\alpha}^{1}(C, I) & \stackrel{f_{*}}{\longleftarrow} & H C_{\alpha}^{1}(C, A) & \longleftarrow & H C_{\alpha}^{1}(C, B)
\end{array}
$$

$$
\begin{array}{ccccc}
H C_{\alpha}^{0}(I, C) & \longleftarrow & H C_{\alpha}^{0}(A, C) & \stackrel{f^{*}}{\longleftarrow} & H C_{\alpha}^{0}(B, C) \\
\downarrow & & & \uparrow \partial \\
H C_{\alpha}^{1}(B, C) & \stackrel{f^{*}}{\longrightarrow} & H C_{\alpha}^{1}(A, C) & \longrightarrow & H C_{\alpha}^{1}(I, C) .
\end{array}
$$

Proof. We refer to [Pu1] for notation. The map $f: A \rightarrow B$ induces the map

$$
f_{*}: X_{*}(\Omega \mathcal{R} A) \rightarrow X_{*}(\Omega \mathcal{R} B)
$$

on $X$-complexes, which is surjective due to the fact that $s$ is a bounded linear section. We denote its kernel by $\left(X_{*}(\Omega \mathcal{R} A), X_{*}(\Omega \mathcal{R} B)\right)$, the relative $X$-complex of the pair $(A, B)$. Then the short sequence

$$
0 \rightarrow\left(X_{*}(\Omega \mathcal{R} A), X_{*}(\Omega \mathcal{R} B)\right) \rightarrow X_{*}(\Omega \mathcal{R} A) \rightarrow X_{*}(\Omega \mathcal{R} B) \rightarrow 0
$$

is exact. So, to prove excision it is enough to replace $\left(X_{*}(\Omega \mathcal{R} A), X_{*}(\Omega \mathcal{R} B)\right)$ by its subcomplex $X_{*}(\Omega \mathcal{R} I)$. This was done for general extensions of Ind-Fréchet algebras with supports in [Pu3]. Indeed, we know that the inclusion

$$
X_{*}(\mathcal{R} I) \hookrightarrow\left(X_{*}(\mathcal{R} A), X_{*}(\mathcal{R} B)\right)
$$

has a homotopy inverse. In order to extend the above result to the differential graded setting we use Theorem 4.14 of [Pu1] and break the discussion into two cases.

Since the kernel of the natural map

$$
H_{\mathrm{Hom}}^{*}\left(X_{*}(\Omega R C), X_{*}(\Omega R-) \otimes V .\right) \rightarrow \operatorname{Hom}_{D G}^{*}\left(X_{*}(\Omega R C), X_{*}(R-) \otimes V .\right)
$$

is null homotopic, (2.1) implies that the chain map

$$
X_{\alpha}^{*}(C, I) \rightarrow X_{\alpha}^{*}(C,(A, B))
$$

is a natural quasi-isomorphism, and consequently excision holds in the second variable. 
Since the kernel of the map

$$
\operatorname{Hom}_{D G}^{*}\left(X_{*}(\Omega R-), X_{*}(\Omega R C)\right) \rightarrow \operatorname{Hom}^{*}\left(X_{*}(R-), X_{*}(R C)\right)
$$

is null homotopic, (2.1) induces a natural quasi-isomorphism as follows:

$$
H_{D o m}^{*}\left(\left(\left(X_{*} \Omega R A\right),\left(X_{*} \Omega R B\right)\right), X_{*}(\Omega R C)\right) \rightarrow H_{D o m}^{*}\left(\left(X_{*} \Omega R I\right), X_{*}(\Omega R C)\right) .
$$

After taking the inductive limit over (2.2) and using the fact that for given admissible Fréchet algebras $E, F$, the map

$$
\lim _{K} \underset{L}{\lim } \operatorname{Hom}_{D G}\left(\bar{X}_{*}\left(\Omega R \tilde{E}_{(K, N)}\right), \bar{X}_{*}\left(\Omega R \tilde{F}_{(L, M)}\right)\right) \rightarrow X_{\alpha}^{*}(E, F)
$$

induces a natural transformation on cohomolgy groups, we conclude that (2.2) induces the following quasi-isomorphism chain map, which implies excision in the first variable:

$$
X_{\alpha}^{*}((A, B), C) \rightarrow X_{\alpha}^{*}(I, C)
$$

Corollary 2.2. Let $A, B$ be two separable $C^{*}$-algebras and $\alpha$ be a (strongly) continuous action of $\mathbb{R}$ on $A$. Then

(1) $H C_{\alpha}^{*}(A, B) \cong H C_{\alpha}^{*+1}(S A, B) \cong H C_{\alpha}^{*+1}(A, S B)$,

(2) $H C_{\alpha}^{*}(A, B) \cong H C_{\alpha}^{*}(S A, S B)$,

(3) $H C_{\alpha}^{*}\left(A \times_{\alpha} \mathbb{R}, B\right) \cong H C_{\alpha}^{*+1}(A, B)$,

(4) $H C_{\alpha}^{*}\left(B, A \times_{\alpha} \mathbb{R}\right) \cong H C_{\alpha}^{*+1}(B, A)$.

Proof. The six-term exact sequences obtained in the previous theorem show that the bivariant asymptotic cyclic cohomology is split exact in both variables, so we can use Theorem 1.2. Now for a given $C^{*}$-algebra $D$ suppose $\alpha_{S D} \in H C_{\alpha}^{1}\left(S^{2} D, S D\right)$ and $\beta_{S D} \in H C_{\alpha}^{1}\left(S D, S^{2} D\right)$ are, respectively, Dirac and Bott elements defined in Definition 9.3 of [Pu1]. Also let $\hat{i}$ be the dual action of the trivial action of $\mathbb{R}$ on D. Then

$$
\begin{aligned}
H C_{\alpha}^{*}(A, B) & \cong H C_{\alpha}^{*}\left(S\left(A \times \times_{\hat{i}} \mathbb{R}\right), B\right) \\
& \cong H C_{\alpha}^{*}\left(S^{2} A, B\right) \\
& \cong H C_{\alpha}^{*+1}(S A, B),
\end{aligned}
$$

where isomorphisms respectively come from Takai duality, Theorem 1.2 and composition by $\beta_{S A}$. Similarly, the second isomorphism of (1) is proved by using $\alpha_{S B}$ on the second variable. From (1), (2) is obvious. For (3) we observe that

$$
\begin{aligned}
H C_{\alpha}^{*}\left(A \times_{\alpha} \mathbb{R}, B\right) & \cong H C_{\alpha}^{*+1}\left(S\left(A \times_{\alpha} \mathbb{R}\right), B\right) \\
& \cong H C_{\alpha}^{*+1}\left(S^{2} A, B\right) \\
& \cong H C_{\alpha}^{*+1}(A, B),
\end{aligned}
$$

and similarly for (4).

Theorem 2.1 and the universal property of $K K$-theory among stable, homotopy invariant, and split exact functors, $[\mathrm{H}$, implies the existence of an unstable ChernConnes character for $C^{*}$-algebras. 
Corollary 2.3. For given $C^{*}$ - algebras $A, B$, there exists a natural transformation

$$
\text { ch: } K K^{*}(A, B) \rightarrow H C_{\alpha}^{*}(A, B),
$$

which is compatible with Kasparov's product of $K K$-theory and the composition product of asymptotic cyclic cohomology.

For the precise meaning of compatibility of products, and to extend the above Chern-Connes character to a larger category of algebras, one can see Theorem 6.3 and Remark 6.5 of [Pu3]. As a consequence of Corollary 2.2, we have the following corollary.

Corollary 2.4. (1) Let $X, Y$ be two finite $C W$-complexes. Then

$$
H C_{\alpha}^{*}(C(X), C(Y)) \cong \underset{\substack{n+m \equiv * \\(\bmod 2)}}{\operatorname{Hom}}\left(\bigoplus_{n=0}^{\infty} H^{n}(X, \mathbb{C}), \bigoplus_{m=0}^{\infty} H^{m}(Y, \mathbb{C})\right),
$$

where $H^{*}(X, \mathbb{C})$ denotes singular cohomology of $X$ with coefficients in $\mathbb{C}$.

(2) Let $X$ be a locally compact metrisable topological space (or equivalently, $C(X)$ is a separable $C^{*}$-algebra). Then

$$
H C_{*}^{\alpha}(C(X))=H C_{\alpha}^{*}(\mathbb{C}, C(X)) \cong \bigoplus_{n=-\infty}^{\infty} H_{c}^{*+2 n}(X, \mathbb{C}),
$$

where $H_{c}^{*}(X, \mathbb{C})$ denotes sheaf cohomology with compact supports and coefficients in $\mathbb{C}$.

Proof. See Theorems 11.2 and 11.7 of [Pu1].

2.2. Local cyclic cohomology. All of the aforementioned results on asymptotic cyclic cohomology can be proved (even easier) for local cyclic cohomology too. Therefore, we bring only some remarks here.

Remarks 2.5. (a) Since we have a natural transformation compatible with composition products from bivariant asymptotic cyclic cohomology to bivariant local cyclic cohomology, all asymptotic cyclic equivalences such as $\alpha_{S-}$ and $\beta_{S_{-}}$, induce similar local cyclic equivalences; see 11.9.b of [Pu1] and 3.23 of [Pu2].

(b) Already there is an unstable excision for local cyclic (co)homology; see Theorem 5.12 of [Pu3]. Therefore, results 2.1 to 2.4 are true for local cyclic theory too.

Since local cyclic cohomology behaves reasonably under inductive limits, Corollary 2.4 is adapted as follows for local cyclic theory:

Corollary 2.6. Let $A, B$ be separable, commutative $C^{*}$-algebras with corresponding locally compact spaces $X, Y$. Then

$$
H C_{l c}^{*}(A, B) \cong \underset{\substack{n+m \equiv * \\(\bmod 2)}}{\operatorname{Hom}}\left(\bigoplus_{n=0}^{\infty} H_{c}^{n}(X, \mathbb{C}), \bigoplus_{m=0}^{\infty} H_{c}^{m}(Y, \mathbb{C})\right) .
$$

Remarks 2.7. (a) Let $\mathcal{C}$ be the class of $C^{*}$-algebras described in Theorem 10.7 of Pu1. For any $A, B$ in $\mathcal{C}$ one obtains the following isomorphisms:

$$
\begin{aligned}
& \text { ch: } K K^{*}(A, B) \otimes_{\mathbb{Z}} \mathbb{C} \stackrel{\cong}{\longrightarrow} H C_{l c}^{*}(A, B), \\
& \text { ch: } K K^{*}(A, B) \otimes_{\mathbb{Z}} \mathbb{C} \stackrel{\cong}{\longrightarrow} H C_{\alpha}^{*}(A, B) .
\end{aligned}
$$


By Proposition 6.2 of [C2] all commutative separable $C^{*}$-algebras belong to $\mathcal{C}$.

(b) As an example we have:

$$
\begin{aligned}
& H C_{*}^{\alpha}\left(C\left(\mathbb{T}^{n}\right)\right) \cong H C_{\alpha}^{*}\left(C\left(\mathbb{T}^{n}\right)\right) \cong \mathbb{C}^{2^{n-1}}, \quad *=0,1, \\
& H C_{*}^{l c}\left(C\left(\mathbb{T}^{n}\right)\right) \cong H C_{l c}^{*}\left(C\left(\mathbb{T}^{n}\right)\right) \cong \mathbb{C}^{2^{n-1}}, \quad *=0,1 ;
\end{aligned}
$$

see $[\mathrm{R} 2]$.

As another tool from $K$-theory, Meyer proved the Universal Coefficient Theorem for bivariant local cyclic cohomology in $[\mathrm{M}]$.

\section{Strict DEFormation quANTIZATiON}

Motivated by formal deformation quantization, Rieffel in [R1] introduced a $C^{*}$ algebraic framework for deformation quantization known as strict deformation quantization. Suppose $A$ is a $C^{*}$-algebra with a dense ${ }^{*}$-subalgebra $\mathcal{A}$ equipped with a Poisson bracket $\{$,$\} . Then a strict deformation quantization of A$ in the direction of $\{$,$\} consists of an open interval I$ containing 0 , and a family of pre$C^{*}$-algebra structures $\left\{\left(\times_{\hbar},{ }^{*},\|\|_{\hbar}\right)\right\}_{\hbar \in I}$ on $\mathcal{A}$ which for $\hbar=0$ coincides with the pre- $C^{*}$-structure inherited from $A$ such that if we denote by $A_{\hbar}$ the completion of $\mathcal{A}$ under the $C^{*}$-structure $\left(\times_{\hbar},{ }^{*}{ },\|\|_{\hbar}\right)$, then the family $\left\{A_{\hbar}\right\}_{\hbar \in I}$ with constant sections from $I$ into $\mathcal{A}$ constitutes a continuous field of $C^{*}$-algebras and for every $a, b \in \mathcal{A}$ we have

$$
\lim _{\hbar \rightarrow 0}\left\|\frac{\left(a \times_{\hbar} b-a b\right)}{i \hbar}-\{a, b\}\right\|=0 .
$$

The first examples of this definition are noncommutative tori and noncommutative Heisenberg manifolds. Deformation quantization of $C^{*}$-algebras by actions of finitedimensional real vector spaces, formulated in [R3], provides other examples for strict deformation quantization. In these constructions, the actions of $\mathbb{Z}$ and $\mathbb{R}$ play important roles. As a consequence, the quantized algebras naturally are associated to appropriate crossed product $C^{*}$-algebras by actions of $\mathbb{Z}$ and $\mathbb{R}$. Thus, the Connes-Thom isomorphism and the Pimsner-Voiculescu exact sequence can be used to compare the $K$-theory of the quantized $C^{*}$-algebra with that of the original $C^{*}$ algebra, R4, A2]. Now, these tools have been generalized for stable, split exact, and homotopy invariant functors. So, we can repeat Rieffel's and Abadie's works to obtain the same results for these functors, in particular for local and asymptotic cyclic (co)homology.

3.1. Noncommutative Heisenberg manifolds. For each positive integer $c$, the Heisenberg manifold $M_{c}$ is defined by the quotient $G / D_{c}$, where $G$ is the Heisenberg group,

$$
G=\left\{\left(\begin{array}{ccc}
1 & y & z \\
0 & 1 & x \\
0 & 0 & 1
\end{array}\right) ; x, y, z \in \mathbb{R}\right\}
$$

and $D_{c}$ is the subgroup of $G$ with $x, y, c z \in \mathbb{Z}$. Every nonzero Poisson bracket on $M_{c}$ is determined by two real numbers $\mu, \nu$, where $\mu^{2}+\nu^{2} \neq 0$. According to A2, deformation quantization of $M_{c}$ in the direction of the nonzero Poisson bracket $(\mu, \nu)$, which is invariant under the action of $G$ by left translation, is denoted by $\left\{D_{\mu \nu}^{c, \hbar}\right\}_{\hbar \in \mathbb{R}}$, where $\hbar$ is the parameter of deformation. We know from $\mathbb{R} 1$, A2 that 
for $\hbar \neq 0$, the algebra $D_{\mu \nu}^{c, \hbar}$ is the generalized fixed-point algebra of $C_{0}(\mathbb{R} \times \mathbb{T}) \times_{\lambda^{\hbar}} \mathbb{Z}$ under the action $\rho$, where

$$
\lambda_{k}^{\hbar}(x, y)=(x+2 k \hbar \mu, y+2 k \hbar \nu), \quad k \in \mathbb{Z}
$$

and if $e(x)=\exp (2 \pi i x)$, then

$$
\left(\rho_{k} \Phi\right)(x, y, p)=e(\operatorname{ckp}(y-\hbar p \nu)) \Phi(x+k, y, p), \quad k \in \mathbb{Z} .
$$

It follows from Theorem 2.11 of [A2] that the algebra $D_{\mu \nu}^{c, \hbar}$ is strongly Morita equivalent to another fixed-point algebra obtained by the action $\gamma^{\lambda^{\hbar}}$ of $\mathbb{Z}$ on $C_{0}(\mathbb{R} \times \mathbb{T}) \times_{\sigma} \mathbb{Z}$, where

$$
\sigma_{k}(x, y)=(x-k, y), \quad k \in \mathbb{Z}
$$

and

$$
\left(\gamma_{p}^{\lambda^{\hbar}} \Phi\right)(x, y, k)=e(-c k p(y-\hbar p \nu)) \Phi(x-2 p \hbar \mu, y-2 p \hbar \nu, k), \quad p \in \mathbb{Z} .
$$

Now, Corollary 1.4 and the homotopy $\hbar \rightarrow \lambda^{\hbar}$ show that local and asymptotic cyclic (co)hom- ology groups of $D_{\mu \nu}^{c, \hbar}$ are independent of the parameter $\hbar$. On the other hand, for any real number $\hbar, D_{\mu \nu}^{c, \hbar}$ and $D_{\hbar \mu, \hbar \nu}^{c, 1}$ are isomorphic. So, one can drop the parameter $\hbar$ from the notation and simply write $D_{\mu, \nu}^{c}$ instead of $D_{\hbar \mu, \hbar \nu}^{c, 1}$. Also, for any pair of integers $k, l, D_{\mu \nu}^{c, \hbar}$ and $D_{\mu+k, \nu+l}^{c, \hbar}$ are isomorphic; see Proposition 1 of [A1]. Thus, the assumption $\hbar \neq 0$ can be ignored. This shows that every stable, homotopy invariant, and split exact (co)functor $F$ is rigid under deformation quantization of Heisenberg manifolds. As a consequence, we have

Theorem 3.1. For any real numbers $\mu, \nu, \hbar$ and integer $c$, even and odd asymptotic and local cyclic homology and cohomology groups of the Heisenberg manifold $D_{\mu \nu}^{c, \hbar}$ are isomorphic to $\mathbb{C}^{3}$.

Proof. It is enough to consider only the commutative case, which is an easy application of Remark 2.7(a) and Theorem 3.4 of [A2].

3.2. Deformation quantization of $C^{*}$-algebras by actions of $\mathbb{R}^{n}$. Let $\alpha$ be a strongly continuous action of $\mathbb{R}^{n}$ on the $C^{*}$-algebra $A$, and let $A^{\infty}$ be the dense *-subalgebra of its smooth vectors. Also, let $J$ be a skew-symmetric matrix on $\mathbb{R}^{n}$. On $A^{\infty}$ the deformed product $\times_{J}$ is defined by

$$
a \times_{J} b=\iint \alpha_{J u}(a) \alpha_{v}(b) e(u \cdot v), \quad a, b \in A^{\infty},
$$

where $e(t)=\exp (2 \pi i t)$. Also, a $C^{*}$-norm \|\|$_{J}$ and an involution $*_{J}$ compatible with the product $\times_{J}$ are defined on $A^{\infty}$ (for details, see [R3]). The completion of this pre- $C^{*}$-structure is denoted by $A_{J}$ and is called the quantization of $A$ by the action of $\alpha$ in the direction of $J$. Let $A$ be separable. Then it is $\sigma$-unital, and consequently $A_{J}$ is $\sigma$-unital too; see [R3]. It was shown in [R4] that $A_{J}$ is strong Morita equivalent to a stable crossed product of $A$ as follows:

$$
A_{J} \stackrel{M}{\simeq} A \times_{\rho} \mathbb{R}^{n} \otimes C_{0}\left(\mathbb{R}^{m}\right) \otimes \mathcal{K}
$$

where $m$ is the dimension of the kernel of $J$. The right side of the above equivalence is separable, so $A_{J}$ is separable too. Thus we have

Theorem 3.2. Local and asymptotic cyclic homology and cohomology of the $C^{*}$ algebra $A$ are rigid under deformation quantization by actions of $\mathbb{R}^{n}$. 
Proof. We consider only asymptotic cyclic homology, others being similar. From the above discussion, Remark 1.5 and Corollary 2.2 we have

$$
H C_{*}^{\alpha}\left(A_{J}\right) \cong H C_{*+m+n}^{\alpha}(A) \text {. }
$$

Since $J$ is a skew-symmetric matrix, $m$ is odd if and only if $n$ is odd, so always $m+n$ is even.

Remarks 3.3. (a) As an example, we consider the $n$-dimensional noncommutative torus $\mathbb{T}_{\theta}^{n}$. Even and odd local and asymptotic cyclic homology and cohomology groups of $\mathbb{T}_{\theta}^{n}$ are isomorphic to $\mathbb{C}^{2^{n-1}}$; see 10.2 of $\mathrm{R} 3$ and Remark 2.7 (b).

(b) Theorems 7.5 of [Pu1] and 3.19 of [Pu2] show that the inclusion $A^{\infty} \rightarrow A$ induces isomorphisms between asymptotic and local cyclic homology and cohomology groups.

(c) Let $\alpha$ also denote the action induced by $\alpha$ on $A_{J}$. The subalgebra of smooth elements of this action is $A^{\infty}$ too; see Theorem 7.1 of [R3]. By part (b), one obtains that the asymptotic and local cyclic (co)homologies of smooth algebras are rigid under deformation quantization too.

\section{ACKNOWLEDGMENT}

I would like to thank Prof. R. J. Stanton, Prof. H. Moscovici and Prof. J. Cuntz for their help and guidance. Without their guidance, it could be very difficult for me to complete this work. Also, I would like to thank the referee whose suggestion helped me to prove theorem 2.1.

\section{REFERENCES}

[A1] ABADIE, B. Vector bundles over quantum Heisenberg manifolds. Algebraic methods in operator theory. Birkhäuser Boston, Boston, MA, 307-315, 1994. MR1284956 (95j:58006)

[A2] ABADIE, B. Generalized fixed-point algebras of certain actions on crossed products. Pacific. J. Math. 171 (1995), no.1, 1-21. MR1362977 (96m:46121)

[AEE] Abadie, B., Eilers, S., Exel, R. Morita equivalence for crossed products by Hilbert $C^{*}$-bimodules. Transactions of the Amer. Math. Soc. 350 (1998), no.8, 3043-3054. MR.1467459 (98k:46109)

[B] BlACKADAR, B. $K$-theory for operator algebras. MSRI Publications, Springer-Verlag, New York, 1986. MR0859867 (88g:46082)

[BGR] Brown, L. G., Green, Ph., Rieffel, M. A. Stable isomorphism and strong Morita equivalence of $C^{*}$-algebras. Pacific J. Math. 71 (1977), no. 2, 349-363. MR0463928 $(57: 3866)$

[C1] CunTZ, J. Generalized homomorphisms between $C^{*}$-algebras and $K K$-theory. Dynamics and processes (Bielefeld, 1981), 31-45, Lecture Notes in Math. 1031, Springer, Berlin, 1983. MR 0733641 (85j:46126)

[C2] Cuntz, J. K-theory and $C^{*}$-algebras. Algebraic K-theory, number theory, geometry and analysis (Bielefeld, 1982), 55-79, Lecture Notes in Math. 1046, Springer, Berlin, 1984. MR.0750677 (86d:46071)

[C3] Cuntz, J. A new look at $K K$-theory. K-Theory 1 (1987), no.1, 31-51. MR0899916 (89a:46142)

[E] Exel, R. Circle actions on $C^{*}$-algebras, partial automorphisms, and a generalized Pimsner-Voiculescu exact sequence. J. Funct. Anal. 122 (1994), no.2, 361-401. MR.1276163(95g:46122)

[FS] Fack, T., Skandalis, G. Connes' analogue of the Thom isomorphism for the Kasparov groups, Inventiones Math. 64 (1981), 7-14. MR0621767 (82g:46113)

[H] Higson, N. A characterization of $K K$-theory, Pacific J. Math. 126 (1987), no. 2, 253276. MR 0869779|(88a:46083) 
[KhS] Khoshkam, M., Skandalis, G. Toeplitz algebras associated with endomorphisms and Pimsner-Voiculescu exact sequences. Pacific J. Math. 181 (1997), no.2, 315-331. MR 1486534 (98k:46088)

[M] Meyer, R. Comparisons between periodic, analytic, and local cyclic cohomology, math.KT/ 0205276.

[NT1] Nest, R., Tsygan, B. Algebraic index theorem, Comm. Math. Phys.172 (1995), 223262. MR 1350407 (96j:58163b)

[NT2] Nest, R., Tsygan, B. Algebraic index theorem for families, Adv. Math. 113 (1995), 151-205. MR1337107 (96j:58163a)

[P] Pimsner, M. A class of $C^{*}$-algebras generalizing both Cuntz-Krieger algebras and crossed products by Z. Free probability theory (Waterloo, 1995), 189-212, Fields Inst. Commun. 12, Amer. Math. Soc. Providence, RI, 1997. MR1426840 (97k:46069)

[PV] Pimsner, M., Voiculescu, D. Exact sequences for $K$-groups and Ext-groups of certain cross-product $C^{*}$-algebras. J. Operator Theory 4 (1980), no.1, 93-118. MR0587369 (82c:46074)

[Pu1] Puschnigg, M. Asymptotic cyclic cohomology. Springer Lecture Notes in Mathematics. 1642 (1996). MR,1482804 (99e:46098)

[Pu2] Puschnigg, M. Cyclic homology theories for topological algebras. K-theory Preprint Archives 292.

[Pu3] PuschnigG, M. Excision in cyclic homology theories. Invent. Math. 143 (2001), 249-323. MR.1835389 (2002e:16014)

[R1] Rieffel, M. A. Deformation quantization of Heisenberg manifolds. Comm. Math. Phys. 122 (1989), no. 4, 531-562. MR1002830 (90e:46060)

[R2] Rieffel, M. A. Noncommutative tori - a case study of noncommutative differentiable manifolds. Geometric and topological invariants of elliptic operators (Brunswick, ME, 1988), 191-211, Contemp. Math. Vol. 105, Amer. Math. Soc., Providence, RI, 1990. MR.1047281 (91d:58012)

[R3] Rieffel, M. A. Deformation quantization for actions of $\mathbb{R}^{d}$. Mem. Amer. Math. Soc. 106 (1993), no. 506, x+93 pp. MR 1184061 (94d:46072)

[R4] Rieffel, M. A. $K$-groups of $C^{*}$-algebras deformed by actions of $\mathbb{R}^{d}$. J. Funct. Anal. 116 (1993), no.1 199-214. MR1237992 (94i:46088)

[Ro1] Rosenberg, J. M. Rigidity of $K$-theory under deformation quantization. q-alg/ 9607021.

[Ro2] Rosenberg, J. M. Behavior of $K$-theory under quantization. Operator algebras and quantum field theory (Rome, 1996), 404-415, Internat. Press, Cambridge, MA, 1997. MR:1491131(99a:46129)

Department of Mathematics, University of Western Ontario, London, Ontario, CANADA N6A 5B7

E-mail address: vshirbis@uwo.ca 